\title{
On the role of the stratosphere in the process of overflow of mesoscale mountains
}

\author{
K. B. Moiseenko \\ Obukhov Institute of Atmospheric Physics, Moscow, Russia
}

Received: 25 January 2005 - Revised: 14 September 2005 - Accepted: 20 September 2005 - Published: 21 December 2005

\begin{abstract}
A 2-D, two- and three-layer stratified airflow over a mountain of arbitrary shape is considered on the assumptions that upstream wind velocity and static stability within each layer are constant (Long's model). The stratosphere is simulated by an infinitely deep upper layer with enhanced static stability.

The analytical solution for the stream function, as well as first (linear) and second order approximations to the wave drag, are obtained in hydrostatic limit $N_{1} L / U_{0} \rightarrow \infty$, where $N_{1}$ is the Brunt-Väsälä frequency in the troposphere, $L$ is a characteristic length of the obstacle, and $U_{0}$ is upstream velocity. The results of numerical computations show the principal role of long waves in the process of interaction between the model layers for a typical mesoscale mountains for which the hydrostatic approximation proves valid in a wide range of flow parameters, in accordance with the earlier conclusions of Klemp and Lilly (1975). Partial reflection of wave energy from the tropopause produces strong influence on the value of wave drag for typical middle and upper tropospheric lapse rates, leading to a quasi-periodic dependance of wave drag on a reduced frequency $k=N_{1} \tilde{H} / \pi U_{0}(\tilde{H}$ is tropopause height) in the troposphere. The flow seems to be statically unstable for $k \geq 2$ for sufficiently large obstacles (whose height exceeds $1 \mathrm{~km}$ ). In this case, vast regions of rotor motions and strong turbulence are predicted from model calculations in the middle troposphere and the lower stratosphere. The model calculations also point to a testify for possible important role of nonlinear effects associated with finite height of the mountain on the conditions of wave drag amplification in the process of overflow of real mountains.
\end{abstract}

Keywords. Meteorology and atmospheric dynamics (Mesoscale meteorology; Waves and tides)

Correspondence to: K. B. Moiseenko

(konst.dvina@mail.ru)

\section{Introduction}

The existence of buoyant force interaction of stably stratified air flow with surface corrugates leads to the generation of wave disturbances which are able to transmit far away from their source. It was found that partial reflection of wave energy by individual layers with different static stability and/or wind velocity can produce a strong influence on the flow field in the free atmosphere (Scorer, 1949; Blumen, 1965; Eliassen and Palm, 1961; Berkshire and Warren, 1970). Some aspects of this problem were considered in many of investigations (see reviews by Queney et al., 1960; Kozhevnikov, 1970; Smith, 1979; Röttger, 2000), principally in the framework of linear models permitting one to account for actual really observed vertical distributions of atmospheric characteristics, including those at large altitudes (Palm and Foldvik, 1960; Berkshire, 1975).

The linear theory, when applied to the atmosphere, may be of limited value, particularly for the following reasons: (i) The results of model calculations (Smith, 1977; Durran, 1986, 1990; Kozhevnikov, 1999), as well as observations (Lilly, 1978; Smith, 1985; Kozhevnikov and Bedanokov, 1998), show that the wind velocity perturbations are not small for many real atmospheric situations, thus disproving the basic assumption of linear theory; (ii) the investigations on the basis of the linear approach were conducted primarily for strongly idealized topography while the mountain shape and height may exert exceptionally strong influence on the flow structure (Gutman, 1969; Smith, 1977; Lilly and Klemp, 1979; Kozhevnikov, 1999).

In the case of large-amplitude motion the initial nonlinear equation of the task can be reduced to the linear one and solved by the well-developed techniques only for a limited class of flows which are of practical interest for the atmospheric tasks (Claus, 1963; Kozhevnikov and Moiseenko, 2004). The most investigated are still the case when upstream wind velocity and static stability do not depend on height (Long's model for compressible fluid) (Long, 1955; Gutman, 1969). This model was used in a number of works 
in which the flows over obstacles with simple geometry were considered (Miles, 1968; Miles and Huppert, 1968; Huppert and Miles, 1969; Lilly and Klemp, 1979).

Extension of Long's model to multi-layer flow with constant static stability within each layer performed by Kozhevnikov and Bedanokov (1993) let us investigate the combined effects of topography and vertical variations of static stability for a number of observed atmospheric events. In particularly, comparison of calculated flow fields with the observations of lee-wave clouds above the Crimean mountains show that the existence of the layers with enhanced static stability can produce a strong effect on the flow field, especially for the region located downwind from the main ridge, where the formation of partially trapped waves of significant amplitude is possible (Kozhevnikov and Bedanokov, 1993, 1998). It was also pointed out that the lower stratosphere can play an important role in such a process. Further investigations revealed that the correct account for the flow characteristics at altitudes $250-50$ mbar is almost as important as the shape of the mountain (Kozhevnikov, V. N, private report). This conclusion is in agreement with the results of nonlinear numerical computations (see, for example, Durran, 1986, 1990), as well as the earlier conclusion of Klemp and Lilly (1975), according to which there exists a strong response of flow field to the conditions of the partial reflection of the wave energy by the tropopause.

The present paper is a logical continuation of the previous works (Kozhevnikov and Bedanokov, 1998; Kozhevnikov and Moiseenko, 2004) aimed at the investigation of the effects dealing with the vertical variation of flow characteristics within a nonlinear approach. The qualitative analysis of wave drag dependence on the flow characteristics are of primary interest in the work. The role of the stratosphere is investigated in the framework of two- and three-layer flow with an exact account of the mountain shape, as well as the "radiation condition" in the upper layer. Such a formulation let us account for a few factors within a rather simple model which play an essential role in the process of overflow of real mountains: shape of the mountain, average wind velocity and existence of an internal dividing surface (tropopause) between regions of the atmosphere with essentially different rates of static stability. Mathematical formulation and a general solution of the task are given in Sect. 2. Theoretical investigation of the solution is conducted under the additional assumption of smoothness of the relief (i.e. that characteristic length $L$ of the obstacle is large compared to Lyra's scale). In this case the explicit analytic solution can be obtained for the stream line field in the hydrostatic limit $L N / U \rightarrow \infty$ by means of asymptotic evaluation of Fourier integral following Miles and Huppert (1969) (Sect. 3). Corresponding formulae for first and second order approximations to the wave drag are obtained in Sect. 4. Some results of numerical calculations for different mountain shapes and flow parameters based on two- and three-layer models of the atmosphere are given in Sect. 5 and Sect. 6, respectively. The basic conclusions are formulated in Sect. 7.

\section{Governing equations and general solution}

We consider a 2-D ( $\tilde{x}, \tilde{z})$, stably stratified, two-layer flow in which the internal dividing surface (tropopause) is situated at the level $\tilde{H}$, and the upstream velocity $U_{0}$ and temperature lapse rates $\gamma_{j}$ within each layer are constant. Henceforth, the indexes $j=1$ and $j=2$ refer to the lower and the upper layer, correspondingly, and the symbol $(\sim)$ is related to dimensional variables whose non-dimensional counterparts are also used. It is supposed that $\gamma_{2} \leq \gamma_{1}$. The horizontal and vertical coordinates are directed downstream and upwards, respectively, and the level $z=0$ coincides with the bottom flat surface. The flow traverses an obstacle of height $\tilde{h}(\tilde{x})$ and a characteristic length $L$ having a finite cross-sectional area.

In present work, the flow in the troposphere is of primary interest. Thus, the kinematic effect of static compressibility approximately can be ignored and we may therefore define a stream function $\psi$ such that the horizontal and vertical components $\tilde{u}, \tilde{w}$ of wind velocity field $\boldsymbol{V}$ are

$\tilde{u}=\frac{\partial \psi}{\partial \tilde{z}}, \quad \tilde{w}=-\frac{\partial \psi}{\partial \tilde{x}}$.

The condition of no upstream influence leads to:

$|\boldsymbol{V}| \rightarrow U_{0}=$ const, $\quad \gamma_{j} \rightarrow-\frac{d \bar{T}_{j}}{d \tilde{z}}$ as $\tilde{x} \rightarrow-\infty$.

The equation of motion, the continuity equation, and equation for conservation of entropy for inviscid stationary flow may be written with use of Boussinesq approximation as

$(\boldsymbol{V} \nabla) \boldsymbol{V}=-\nabla \pi^{\prime}+\beta T^{\prime} \nabla \tilde{z}$,

$(\boldsymbol{V} \nabla) T^{\prime}=-S \tilde{w}$,

$\nabla \boldsymbol{V}=0$,

$\nabla=\left(\frac{\partial}{\partial \tilde{x}}, \frac{\partial}{\partial \tilde{z}}\right), \quad \beta=\frac{g}{T_{a}}, S=\gamma_{d}-\gamma$,

$\frac{\partial \bar{p}}{\partial \tilde{z}}=-\bar{\rho} g, \quad p=\rho R T, \quad \pi^{\prime}=R T_{a} p^{\prime} / \bar{p}$,

where $g$ is gravitational acceleration, $R$ is gas constant for dry air, $T$ is absolute temperature, $\gamma_{d}$ is dry adiabatic lapse rate, $T_{a}$ is an average temperature within the given layer, $p$ is pressure, and $\rho$ is density (Gutman, 1969). Here $\left(^{\prime}\right)$ represents a local deviation of a given variable from its background (undisturbed) value (which marked with overbar) at an altitude $\tilde{z}$.

Let $\tilde{\delta}$ be the vertical displacement of a streamline from its undisturbed height $z_{0}$ :

$$
\begin{aligned}
\tilde{\delta}(\tilde{x}, \tilde{z}) & =\tilde{z}-\tilde{z}_{0}(\tilde{x}, \tilde{z})=-\psi^{\prime} / U_{0}, \\
\psi^{\prime} & =\psi(\tilde{x}, \tilde{z})-U_{0} \tilde{z} .
\end{aligned}
$$

The proper dimensionless task parameters may be chosen as

$d=\frac{N_{1} \tilde{h}_{\max }}{U_{0}}, k=\frac{H}{\pi}=\frac{2 \tilde{H}}{\lambda_{c 1}}, \varepsilon=\frac{L N_{1}}{U_{0}}, X=\frac{\lambda_{c 1}}{\lambda_{c 2}}=\frac{N_{2}}{N_{1}}$, 
where $\quad N_{j}=\sqrt{\beta_{j} S_{j}} \quad$ is Brunt-Väsälä frequency, $\lambda_{c j}=2 \pi U_{0} / N_{j}$ is Lyra's scale (Lyra, 1943) in the layer $j$. The other dimensionless variables are defined as follows:

$$
\begin{array}{r}
x=\tilde{x} / L,(z, H)=(\tilde{z}, \tilde{H}) N_{1} / U_{0},(u, w) \\
=(\tilde{u}, \tilde{w}) / U_{0},(h, \delta)=(\tilde{h}, \tilde{\delta}) / \tilde{h}_{\max } .
\end{array}
$$

It was shown by Gutman (1969) that the initial set of Eqs. (3-5) under the assumptions formulated above may be reduced to the corresponding Helmholtz's equation for $\delta$ field in the given layer:

$\varepsilon^{-2} \frac{\partial^{2} \delta_{j}}{\partial x^{2}}+\frac{\partial^{2} \delta_{j}}{\partial z^{2}}+D_{j}^{2} \delta_{j}=0, \quad D_{1}=1, D_{2}=X$.

The lower boundary condition is

$\delta(x, d h)=h(x)$.

On the dividing streamline $z_{0 d}(x, z)=H$, the lower and upper flows must satisfy the kinematic condition and the dynamic requirement $p_{1}=p_{2}$, which implies

$\delta_{1}=\delta_{2}, \quad \frac{\partial \delta_{1}}{\partial z}=\frac{\partial \delta_{2}}{\partial z}, \quad$ at $\quad z=H+\zeta(x)$,

where $\zeta(x)$ is a deviation of $z_{0 d}$ from its original height $H$ (Kozhevnikov and Bedanokov, 1993). As it follows from the model calculations, the condition $|\zeta| \ll H$ holds for typical atmospheric values of $U_{0}$ and $N_{j}$. This suggests that $\zeta(x)$ may be set approximately to zero in Eq. (13), by analogy with the previous works (Kozhevnikov and Bedanokov, 1993; Kozhevnikov and Moiseenko, 2004).

Using integral Fourier transform, we can write a solution of Eq. (11) in the form (see Kozhevnikov and Bedanokov, 1993, for details):

$\delta_{j}(x, z)=\int_{-\infty}^{+\infty} f\left(x^{\prime}\right) P_{j}(\xi, z) \mathrm{d} x^{\prime}, \quad \xi=x-x^{\prime}$,

$P_{j}(\xi, z)=\frac{\varepsilon}{\pi} \operatorname{Re} \int_{0}^{+\infty} \varphi_{j}(s, z) e^{i s \varepsilon \xi} \mathrm{d} s$

where $f(x)$ is the equivalent dipole density of the obstacle, $R e$ denotes the real part of integral, and $\varphi_{j}(s, z)$ are the general solutions of the corresponding ordinary differential equations written in the form

$\varphi_{1}(s, z)=C_{1}(s) \sin m_{1} z+\cos m_{1} z$

$\varphi_{2}(s, z)=C_{2}(s) e^{+i m_{2}(z-H)}$,

where $m_{j}=\sqrt{D_{j}^{2}-s^{2}}$. The function $\varphi_{2}(s, z)$ satisfies the radiation condition for long waves, when $m_{2}$ is real, and bounded, when $m_{2}$ is imaginary. Invoking the boundary condition Eq. (13) and Eqs. (14, 16, 17), we obtain

$C_{1}=\frac{\sin \phi_{1}+i X_{1} \cos \phi_{1}}{\cos \phi_{1}-i X_{1} \sin \phi_{1}}, C_{2}=\frac{1}{\cos \phi_{1}-i X_{1} \sin \phi_{1}}$ where $\phi_{1}=m_{1} H_{1}$, and $X_{1}=m_{2} / m_{1}$. Substitution of Eq. (14) into Eq. (12) gives the integral equation

$h(x)=\int_{-\infty}^{+\infty} f\left(x^{\prime}\right) P_{1}\left(x-x^{\prime}, d h\right) \mathrm{d} x^{\prime}$,

which should be solved for $f(x)$. If $d$ is small compared to the unity and $d h / d x$ is uniformly bounded, the lower boundary condition can be linearized by setting $d=0$ in Eq. (12) to obtain

$\delta(x, 0)=h(x)$.

In this case, letting $d h \rightarrow 0$ in Eq. (19) and invoking Eq. (16), we obtain

$P_{1}(\xi, d h) \rightarrow \delta_{0}(\xi), f(x) \rightarrow h(x)(d h \rightarrow 0)$,

where $\delta_{0}$ is the Dirac delta function. Thus the approximate solution of Eq. (19) is $f(x) \approx h(x)$ provided $d \ll 1$. This feature allows an effective numerical solution of Eq. (19) in the case $d \leq 1$ by direct inversion of the corresponding matrix operator, based on the "weak regularization" technique, as described by Kozhevnikov and Moiseenko (2004).

\section{Long-wave limit}

We now suppose that the characteristic length of the obstacle is large compared to the characteristic vertical wavelength, which is close to Lyra's scale. Invoking Eq. (9), the condition of smoothness can be expressed as

$\frac{d \tilde{h}}{d \tilde{x}} \approx \frac{\tilde{h}_{\max }}{L}=d \varepsilon^{-1} \ll 1$, or $\varepsilon \gg 1$ with $d$ fixed.

The asymptotic representation of the general solution Eqs. $(14,15)$ as $\varepsilon \rightarrow \infty$ can be obtained by means of a technique similar to that one described by Miles and Huppert (1969). Replacing $x$ by the complex variable $x_{1}=x+i x_{i}$ in Eq. (15) and integrating by parts with respect to $s$, we obtain:

$$
\begin{aligned}
& P_{j}\left(x_{1}-x^{\prime}, z\right)=\tilde{R} e\left\{i\left(x_{1}-x^{\prime}\right)^{-1} \varphi_{j}(0, z)\right\} / \pi+O\left(\varepsilon^{-1}\right) \\
& \left(\varepsilon \rightarrow \infty, x_{i} \rightarrow 0+\right) .
\end{aligned}
$$

Substituting Eq. (23) into Eq. (14), we obtain the approximation:

$\delta_{j}(x, z)=\tilde{R} e\left\{\varphi_{j}(0, z) f_{1}\left(x_{1}\right)\right\} \quad\left(x_{i} \rightarrow 0+\right)$,

where $f_{1}\left(x_{1}\right)$ is the Cauchy integral of $f(x)$, as defined by

$f_{1}\left(x_{1}\right)=\frac{1}{i \pi} \int_{-\infty}^{+\infty} \frac{f\left(x^{\prime}\right) \mathrm{d} x^{\prime}}{x^{\prime}-x_{1}}\left(x_{i}>0\right)$.

Under the assumptions formulated by Miles and Huppert (1969), the following relation holds:

$f_{1}\left(x_{1}\right)=f(x)-i f^{*}(x)\left(x_{i}=0+\right)$,

$f^{*}(x)=\frac{1}{\pi} P \int_{-\infty}^{+\infty} \frac{f\left(x^{\prime}\right)}{x^{\prime}-x} \mathrm{~d} x^{\prime}$, 
where $P$ is the Cauchy principal value of the integral, and the upper index $(*)$ denotes henceforth the Hilbert transform of the given function (Titchmarsh, 1948). Substitution of Eq. (26) into Eq. (24) gives:

$\delta_{j}(x, z)=\varphi_{j r}(0, z) f(x)+\varphi_{j i}(0, z) f^{*}(x)$

(henceforth, the subscripts $r$ and $i$ the denote real and imaginary parts of the quantity). Using Eqs. $(16,17,28)$, we obtain:

$$
\begin{gathered}
\delta_{1}(x, z)=f(x)\left\{\cos z+C_{1 r} \sin z\right\}+f^{*}(x) C_{1 i} \sin z, \\
\delta_{2}(x, z)=f(x)\left\{C_{2 r} \cos \phi-C_{2 i} \sin \phi\right\} \\
+f^{*}(x)\left\{C_{2 i} \cos \phi+C_{2 r} \sin \phi\right\},
\end{gathered}
$$

where $\phi=X(z-H)$, and $C_{j r}, C_{j i}$ are taken at $s=0$ according to Eq. (18):

$$
\begin{aligned}
& C_{1 r}=-0.5\left(X^{2}-1\right) \sin (2 H) \cdot \Delta^{-1}, \quad C_{1 i}=X \cdot \Delta^{-1}, \\
& C_{2 r}=\cos H \cdot \Delta^{-1}, \quad C_{2 i}=X \sin H \cdot \Delta^{-1}, \\
& \Delta=1+\left(X^{2}-1\right) \sin ^{2} H .
\end{aligned}
$$

It follows from Eqs. (31-33), that if $X \geq 1$ then the value of $\Delta$ is always positive and a solution in the form Eqs. $(29,30)$ exists for any model parameters, for which the conditions formulated at the beginning of page 2 hold.

Setting $\gamma_{1}=\gamma_{2}(X=1)$ in Eqs. $(31,33)$, we obtain $C_{1 r}=0$, $C_{1 i}=1$, and a solution for the one-layer flow in a half space directly follows from Eq. (29):

$\delta(x, z)=f(x) \cos z+f^{*}(x) \sin z$.

This equation is equivalent to the "low-speed limit" approximation by Miles and Huppert (1969), Eq. (5.5b), and reduces to Eq. (1) from (Drazin and Su, 1975) in the planar approximation Eq. (20). Letting $X \rightarrow \infty$ in Eqs. $(29,31,33)$, we obtain

$\delta(x, z)=h(x) \frac{\sin (H-z)}{\sin (H-d h(x))}$,

which is a classical solution of Long's task for a channel of finite height $H$ in a long-wave limit (Long, 1955).

Substitution of Eq. (29) into Eq. (12) gives the singular integral equation

$h(x)=f(x)\left\{\cos (d h)+C_{1 r} \sin (d h)\right\}+f^{*}(x) C_{1 i} \sin (d h)$,

which can be solved for $f$ by standard numerical techniques (Verlan and Sizikov, 1978). The approximate solution for the case $d \leq 1$ can be obtained by expansion of $f(x)$ in a power of $d$ as $f=f^{(0)}+d f^{(1)}+\ldots$. Substituting the expansion into Eq. (36) and separating by order of $d$, we obtain:

$f=h\left(1-d\left(C_{1 r} h+C_{1 i} h^{*}\right)\right)+O\left(d^{2}\right)$.

The errors in relief reproduction associated with use of Eq. (37) can be estimated by computation of the displacement height of zero stream line $z_{0}(x, d h(x))=0$ by means of Eqs. $(29,37)$ and comparing the result with the prescribed contour $h(x)$. It was found that the first and second order approximations given by Eq. (37) produce a relative error in relief reproduction of less than $\pm 5 \%$ and $\pm 15 \%$, correspondingly, in the range $0.3 \leq d \leq 0.8$, for the examples considered below.

\section{Wave drag}

By definition, the exact value of the wave drag of the mountain on the atmosphere is given by

$D_{W}=-\int_{Q} p^{\prime}(\tilde{x}, \tilde{z}) \mathrm{d} \tilde{h}$,

where the integral must be calculated along a contour $Q(\tilde{x}, \tilde{h})$. We also define a wave drag coefficient as

$C_{D}=-D_{W} /\left(0.5 \tilde{h}_{\max } \rho_{0} U_{0}^{2}\right), \rho_{0}=\bar{\rho}(0)$.

The pressure perturbation $p^{\prime}(\tilde{x}, \tilde{h})$ at the lower boundary can be found by means of the Bernoulli equation for Eqs. (3-4) applied to zero streamline. Following Gutman (1969), we have

$\pi^{\prime}=p^{\prime} / \bar{\rho}=-\left(\tilde{u}^{2}+\tilde{w}^{2}-U_{0}^{2}\right) / 2-\beta T^{\prime 2} /(2 S)$,

where $T^{\prime}=-S \tilde{h}, \bar{\rho}=\bar{p}\left(R T_{c}\right)^{-1}$. For the sake of simplicity, we shall replace $\bar{\rho}(\tilde{h})$ by $\rho_{0}$ in Eq. (40), based on the earlier conclusion that such a simplification does not lead to any appreciable errors in wave drag computations, when $\tilde{h}_{\max } \leq 1 \mathrm{~km}$ (Kozhevnikov, 1999). Substituting Eq. (40) into Eq. (38), invoking Eq. (8), and substituting the result into Eq. (39), we obtain

$C_{D}=d \int_{Q}\left\{2 \delta_{z}-d \delta_{z}^{2}-d \varepsilon^{-2} \delta_{x}^{2}\right\} \mathrm{d} h$,

$\lim _{\varepsilon \rightarrow \infty} C_{D}=C_{D H}=d \int_{Q}\left\{2 \delta_{1 z}-d \delta_{1 z}^{2}\right\} \mathrm{d} h$,

where subscripts $x, z$ denote partial derivatives, and $\delta_{1 z}$ in Eq. (42) should be calculated from Eq. (29). Expanding $\delta_{1 z}$ in a powers of $d$ with account of Eqs. $(29,37)$ and substituting the result into Eq. (42), we obtain an approximation

$C_{D H}=d C_{1 i} \Phi_{1}+d^{2}\left(C_{1 r} C_{1 i} \Phi_{2}+C_{1 i}^{2} \Phi_{3}\right)+O\left(d^{3}\right)(d \rightarrow 0)$,

where

$$
\begin{gathered}
\Phi_{i}=-2 \int_{Q} g_{i} \mathrm{~d} h, i=1,2,3, g_{1}=-h^{*}, g_{2}=2 h^{*} h+\left(h^{2}\right)^{*}, \\
g_{3}=\left(h^{*}\right)^{2} / 2+\left(h^{*} h\right)^{*} .
\end{gathered}
$$

If $d \ll 1$, then only the leading term in Eq. (43) should be retained for which the notation $C_{D H}^{(0)}$ will be used. Invoking Eqs. $(9,31,43)$, we obtain the following representations:

$$
\begin{aligned}
& C_{D H}^{(0)}=\frac{\tilde{h}_{\max }}{\lambda_{c 2} \Delta} 2 \pi \Phi_{1}, \\
& =\frac{d X \Phi_{1}}{1+\left(X^{2}-1\right) \sin ^{2}(\pi p d)}, p=\frac{\tilde{H}}{\pi \tilde{h}_{\max }} .
\end{aligned}
$$

For the particular case $X=\Delta=1$, the Eqs. $(45,46)$ are identical to the formulae obtained by Drazin and Su (1975), Eq. (17), and Lilly and Klemp (1979), Eq. (33). 
Using Eq. (46), we can consider two different cases having practical interest. Setting $d$ and $X$ to constants in Eq. (46) and considering $C_{D H}^{(0)}$ as a function of $k=p d$, we conclude from Eqs. $(9,33)$ that the maximal and minimal values of drag coefficient are attained, when the tropopause height satisfies the conditions

$k=K$, or $\tilde{H}_{\max }^{(0)}=K \lambda_{c 1} / 2$

and

$k=K-1 / 2$, or $\tilde{H}_{\min }^{(0)}=(2 K-1) \lambda_{c 1} / 4$,

correspondingly, where $K=1,2, \ldots$. The local extremes of drag coefficient Eq. (45) are given by

$C_{\mathrm{DH} \max }^{(0)}=\frac{\tilde{h}_{\max }}{\lambda_{c 2}} 2 \pi \Phi_{1}$ at $k=K$,

$C_{\mathrm{DLmin}}^{(0)}=\frac{\tilde{h}_{\max }}{\lambda_{c 2} X^{2}} 2 \pi \Phi_{1}$ at $k=K-1 / 2$,

i.e.

$C_{\mathrm{DLmax}}^{(0)} / C_{\mathrm{DLmin}}^{(0)}=X^{2}$.

As it follows from the above given relations, the maximal (minimal) value of $C_{D H}^{(0)}(p)$ in a two-layer flow equals to $\left(X^{2}\right.$ times less than) the corresponding value in a one-layer flow, when $\gamma=\gamma_{2}$. Thus, Eqs. (47-50) reveal a well-known property of the hydrostatic solution in the limit $d \rightarrow 0$ which deals with a local increase (decrease) of flow disturbances, when the wave components responsible for upward and downward energy fluxes are in phase (anti-phase), respectively (Klemp and Lilly, 1975).

The effect of finite height of the obstacle on the conditions of partial resonance can be estimated by differentiation Eq. (43) with respect to $p$ and evaluation of the roots of the resulting equation by means of asymptotic expansion (Olver, 1974). The corresponding values of $k$ and $\tilde{H}$ at which extremal values of drag coefficient are attained can be written as:

$$
\begin{aligned}
k= & K+\kappa d+O\left(d^{2}\right), \tilde{H}_{\max }=\tilde{H}_{\max }^{(0)}+\pi \kappa \tilde{h}_{\max } \\
& +O\left(2 \pi^{2} \tilde{h}_{\max }^{2} / \lambda_{c 1}\right),
\end{aligned}
$$

$k=K-1 / 2+\kappa d+O\left(d^{2}\right), \tilde{H}_{\min }=\tilde{H}_{\min }^{(0)}+\pi \kappa \tilde{h}_{\max }$

$$
+O\left(2 \pi^{2} \tilde{h}_{\max }^{2} / \lambda_{c 1}\right)
$$

where $\kappa=\left|\Phi_{2}\right| / 2 \pi \Phi_{1}>0$. Thus, the nonlinear effects associated with the finite height of the mountain reveal itself in higher values of $k$ at which the local extremes of wave drag are attained compared to those predicted by linear analysis, and such a shift is proportional to the dimensionless height $d$. For the examples considered below $\pi \kappa \approx 1$. It then follows from Eqs. $(52,53)$ that the equivalent increase in the optimal height of the tropopause is of the order of the maximal

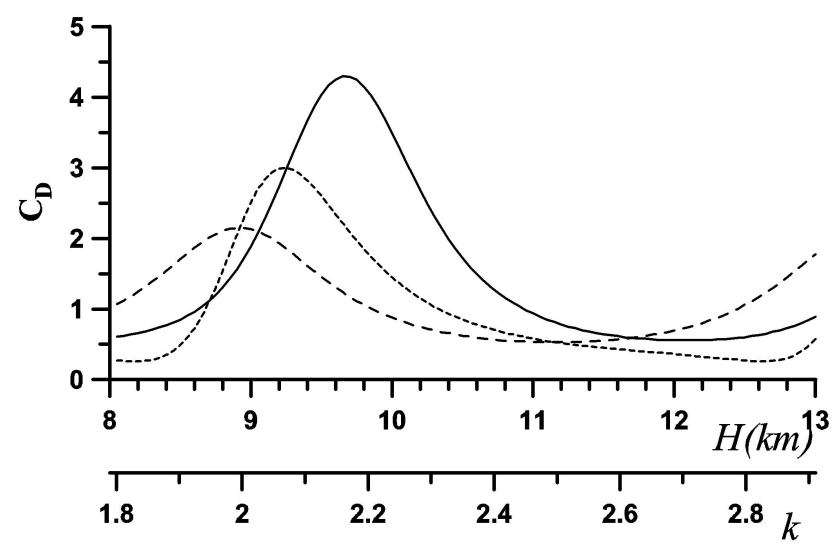

Fig. 1. The first order (long dashed) and second order (dashed) approximations to the wave drag for the Witch of Agnesi profile, as given by Eq. (45) and Eq. (43), relative to the hydrostatic value (solid), as given by Eq. (42).

height of the mountain, i.e. can be rather significant in many real situations.

As an example, the first and second order approximations given by Eq. (43) are compared with the nonlinear solution of Eq. (42) in Fig. 1 for the Witch of Agnesi profile $\tilde{h}=\tilde{h}_{\max } /\left[1+(\tilde{x} / b)^{2}\right]\left(\tilde{h}_{\max }=1 \mathrm{~km}, b=10 \mathrm{~km}\right)$ for $X=2$, $d=0.7, p=2.55 \div 4.14(k=1.79 \div 2.91)$, which corresponds to the upstream velocity $U_{0}=15 \mathrm{~ms}^{-1}$, or $\lambda_{c 1}=8.92 \mathrm{~km}$, and the tropopause height range $8 \div 13 \mathrm{~km}$, with the other dimensional parameters, as in Eq. (54). The given range of $k$ covers the second quasi-resonant peak $(K=2)$ and following minimum $(K=5 / 2)$ of the curve $C_{D H}^{(0)}(k)$. One can see from the figure that the nonlinear drag coefficient (solid curve) attains its local maximum at $\mathrm{H}=9.65 \mathrm{~km}$, i.e. about $750 \mathrm{~m}$ higher if compared with the value predicted by linear analysis (long dashed curve).

The similar conclusions follow from the consideration of the drag coefficient as a function of $d$ with $p$ and $X$ fixed. The local extremes of the function are expressed in terms of the equivalent value of $k$ as described above. In particular, the conditions of local extremes of $C_{D H}^{(0)}$ in this case are defined by the relations whose leading terms are given by Eqs. $(47,48)$ while the other terms have $O\left(1 / 2 \pi^{2} k\left(X^{2}-1\right)\right)$, i.e. can be neglected for typical atmospheric values of $X$. According to Eq. (46), the extremal values of $C_{D H}^{(0)}$ in this case will be proportional to $d$. Calculations suggest that local extremes of the nonlinear drag coefficient are attained at higher values of $k$ (smaller background wind velocities) relative to the extremes of $C_{D H}^{(0)}$, as seen in Figs. 3a-c for the examples considered below, and such a shift is proportional to the values of $k$ and $\tilde{h}_{\max }$. 


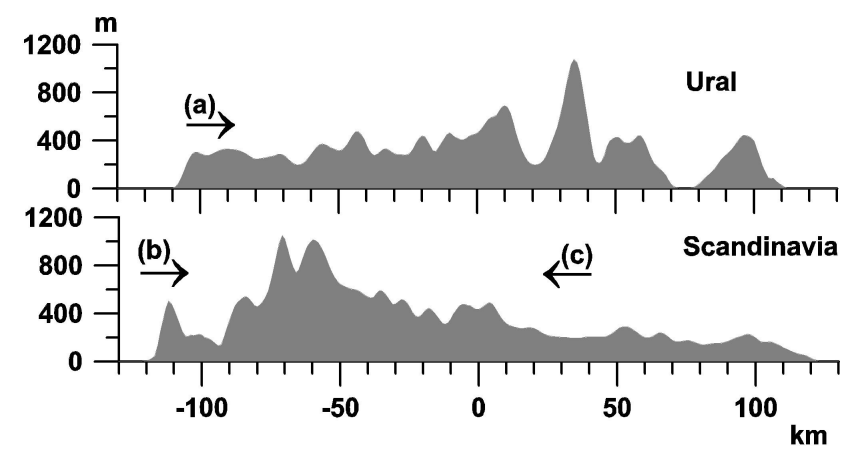

Fig. 2. The idealized terrain shapes for Ural and Scandinavian mountains used in calculations. The flow direction for each of the cases (a-c) considered in Sect. 5 is marked with an arrow.

\section{Examples of calculations for various mountain pro- files}

In this section, the results of nonlinear calculations of some flow characteristics obtained from use of both nonhydrostatic and hydrostatic models described above are shown and compared with each other. The mountain profiles considered below are the meridional cross section of the Ural Mountains at $60^{\circ} \mathrm{N}$, with flow direction from $\mathrm{W}$ to $\mathrm{E}$ (case a), and a NW-SE cross section of the Scandinavian mountains for the region to the north west from Kiruna approximately at $68^{\circ} \mathrm{N}$. Because of its strong asymmetry, two cases were considered for the latter profile, where background flow is from NW (case b) and from SE (case c). These mountain regions were chosen due to their quasi-2-D orography, as well as enhanced wave activity observed frequently during field observations (see Enell et al., 1999; Stebel et al., 2000; Kozhevnikov, 1999). The profiles obtained by some averaging procedure with $1-\mathrm{km}$ horizontal resolution are shown in Fig. 2. For all the cases the value of $\tilde{h}_{\max }$ is close to $1100 \mathrm{~m}$.

The drag coefficients and wave drag for the cases a-c are plotted in Figs. 3a-c and Figs. 4a-c, respectively, as a functions of $k$ for $0.8 \leq k \leq 4, X=2, p=2.9$ which corresponds to dimensional flow parameters

$\gamma_{1}=7 \mathrm{~K} / \mathrm{km}, \quad \gamma_{2}=0 \mathrm{~K} / \mathrm{km}, \quad \tilde{H}=10 \mathrm{~km}, \quad T_{a 1}=240 \mathrm{~K}$

$T_{a 2}=210 \mathrm{~K}, \quad U_{0}=42.0 \div 8.4 \mathrm{~ms}^{-1}, \quad \lambda_{c 1}=25.0 \div 5.4 \mathrm{~m}(54)$

The given range of $k$ comprises the first three peaks of the wave drag (henceforth, the peaks are numerated in ascending order of $k$ ). According to non-hydrostatic solutions, maximal values of the wave drag vary from $3.5 \cdot 10^{5}$ to $6 \cdot 10^{5} \mathrm{H} / \mathrm{m}$ for the case $b$ and from $8 \cdot 10^{5}$ to $11.5 \cdot 10^{5} \mathrm{H} / \mathrm{m}$ for the cases $\mathrm{a}-\mathrm{c}$. The latter values significantly exceed the upper boundary value of $7.5 \cdot 10^{5} \mathrm{H} / \mathrm{m}$, obtained for the much higher St. Gotthard section of the Alps during the ALPEX project (Davies and Phillips, 1985), as well as some model estimations of the wave drag for the Ural Mountains (Kozhevnikov, 1999). Obviously, other accompanying factors, such as upstream lowlevel blocking/deflection and viscous effects, play an important role in real atmospheric situations.
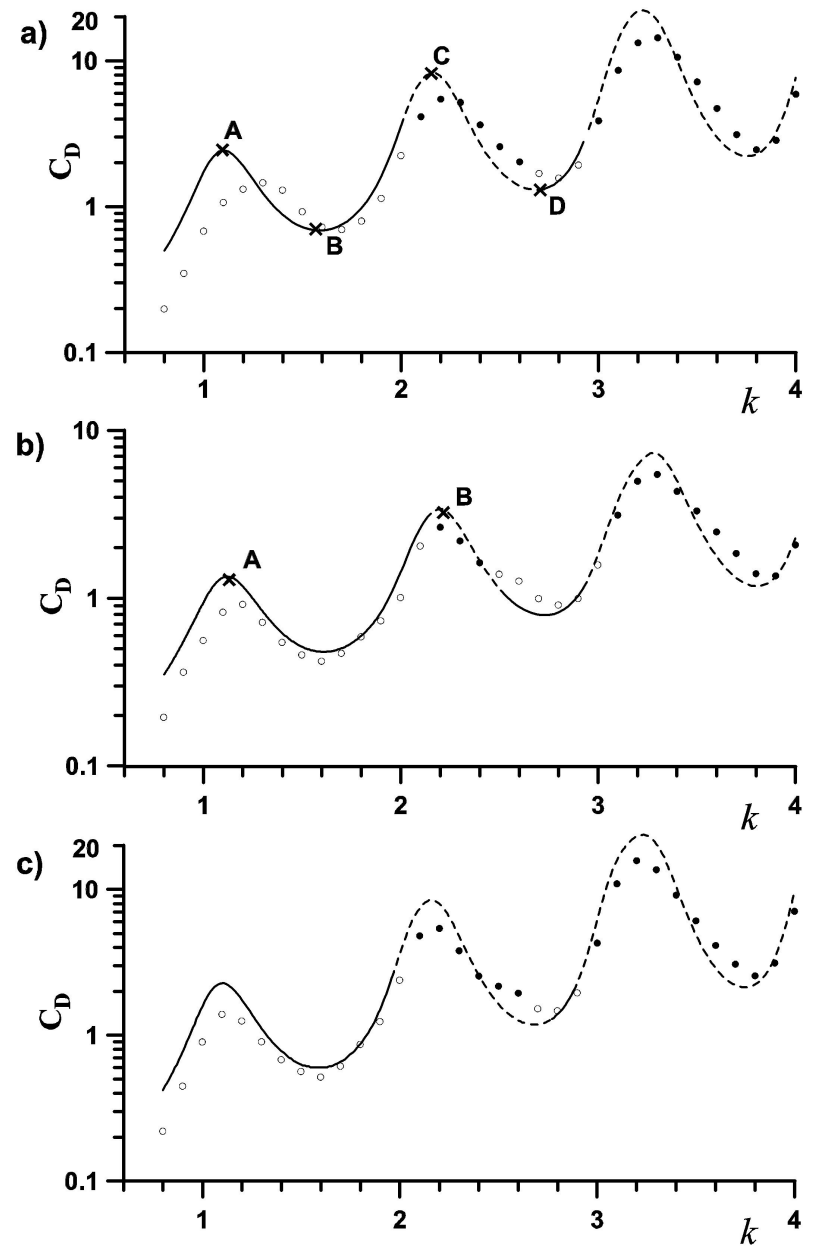

Fig. 3. Drag coefficient as a function of $k$ for (a), Ural, and (b), (c), Scandinavian mountains. Figures $(\mathrm{a}-\mathrm{c})$ correspond to the mountain profiles $(\mathrm{a}-\mathrm{c})$ shown in Fig. 2. The curves and circles are for the hydrostatic and non-hydrostatic solutions, correspondingly. The dashed curves and open circles mark the regions of $k$ over which Long's condition for a statically stable flow, Eq. (55), is violated. The points A-D in Fig. 3a and A, B in Fig. 3b correspond to the flow fields presented in Figs. 5a-d and Figs. 5a and b.

The results plotted in Figs. 4a-c reveal that the values of the wave drag are significantly (a 2-3 times) larger for the profiles with steep leeward slopes (cases a, c), comparing with case $b$, in agreement with the conclusions of Lilly and Klemp (1979). It also follows that sharp, smallscale orography irregularities for the case of a steep windward slope (case b) produce less effect on the flow, comparing to the opposite cases a, c. As a sequence, the flow for case $\mathrm{b}$ is nearly hydrostatic for the whole range of $k$ considered, whereas the use of hydrostatic approximation in the other cases leads to a relatively stronger overestimation of the wave drag values in the vicinities of the quasi-resonant peaks and at small values of $k$, due to the inability to account properly for the short waves produced by the obstacle. On the other hand, the use of the linear approximation Eq. (45) 
Table 1. The ranges of $k$ and $d$ in which the flow is statically unstable, with the corresponding bound values of drag coefficient.

\begin{tabular}{lcrr}
\hline (a) North Ural & $k$ & $2.02-2.70$ & $\geq 2.95$ \\
W-E & $d$ & $0.68-0.91$ & $\geq 1.00$ \\
& $C_{D H}$ & $4.45 \rightarrow 1.31$ & 3.39 \\
(b) Scandinavia & $k$ & $2.14-2.51$ & $\geq 2.95$ \\
NW-SE & $d$ & $0.70-0.83$ & $\geq 0.98$ \\
& $C_{D H}$ & $3.10 \rightarrow 1.10$ & 1.30 \\
(c) Scandinavia & $k$ & $1.97-2.76$ & $\geq 2.89$ \\
SE-NW & $d$ & $0.65-0.91$ & $\geq 0.95$ \\
& $C_{D H}$ & $2.73 \rightarrow 1.27$ & 2.15 \\
\hline
\end{tabular}

leads to an overestimation of the drag coefficient for $k<1.1$ $\left(U_{0} \geq 30 \mathrm{~ms}^{-1}\right)$ and an underestimation at the higher values of $k$ (lower wind velocities), comparing to the non-hydrostatic solution.

As seen from Figs. 3 and 4, the model calculations predict two ranges of $k$ and $d$ in which Long's condition of static stability

$d \frac{\partial \delta_{1}}{\partial z}<1$

is violated, i.e. the closed streamlines (rotors) exist in the flow field. These ranges, as well as the corresponding bound values of $C_{D H}$ are presented below. These ranges, as well as the corresponding bound values of $C_{D H}$ are presented in Table 1 .

Here the right arrow shows the decrease in the drag coefficient when moving from the left to right boundary of the first range located near the second peak between approximately $k=2$ and $k=2.7$. (This range is slightly shifted to the higher values of $k$ relative to the center of the peak at $k \approx 2.2$ because the increase in the corresponding value of $d$, along with $k$, produces more favorable conditions for overturning.) As follows from Eq. (9), the lower boundary values $k=2.0 \div 2.17$ correspond to the flow parameters, for which the vertical wave length becomes equal to or less than the tropopause height $\tilde{H}$. As it can be seen, the flow is unstable for $k \geq 3.0$ in all the cases considered. According to the calculations, the ranges of $k$ associated with unstable flow in the stratosphere (not shown here) are close to those for the troposphere, although somewhat more extensive because the enhanced static stability provides more favorable conditions for overturning (Miles and Huppert, 1968; Klemp and Lilly, 1978).

Figures $3 a-c$ and $4 a-c$ also show a good quantitative agreement between the phases of curves, as well as boundary values of $k$ and $d$ for three types of the obstacles which are essentially different in their shapes. According to the table the lower bound values of $d$ for the first range of $k$ where the flow is unstable are close to each other for the cases a-c, as well as to the limiting boundary value $k=0.67$ obtained by Miles and Huppert (1968), for a semi-elliptical obstacle as
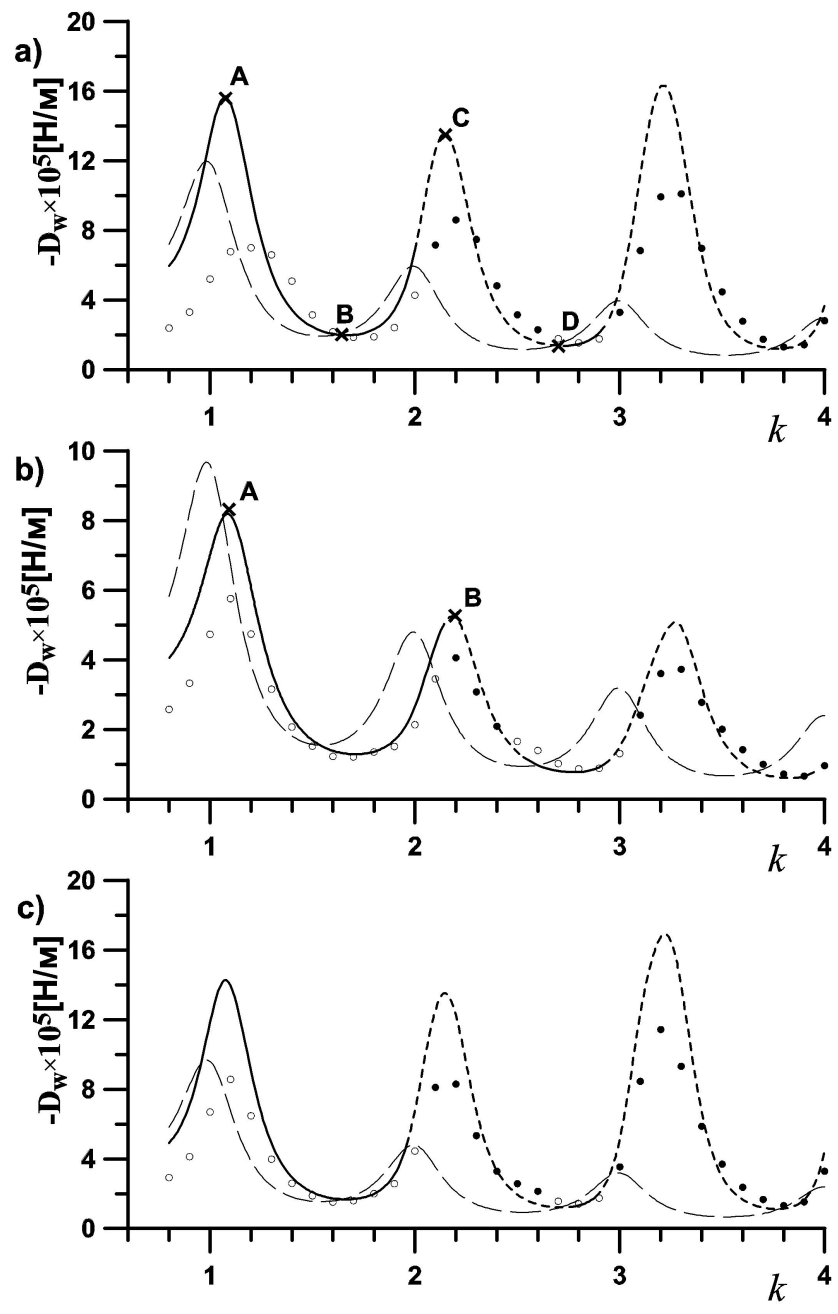

Fig. 4. Wave drag as a function of $k$ for (a), Ural, and (b), (c), Scandinavian mountains. Notations are the same as in Fig. 3. For each case, the results for $D_{W}$, based on the linear drag coefficient, Eq. (45), are also plotted as dashed curves.

$\varepsilon \rightarrow \infty$. Thus the basic flow characteristics in the hydrostatic limit proves to be almost insensitive to individual small-scale peculiarities of the relief, i.e. in agreement with the earlier conclusion of Smith (1977).

The quasi-periodical response to the tropospheric value of the reduced frequency reveals itself distinctly in a flow field. Figures 5a-d show the streamlines over the Ural Mountains for the first two local maxima (Figs. 5a and c) and minima (Figs. 5b and d) of the curve $C_{D H}(k)$ plotted in Fig. 3a. Flows in Figs. 5a-d correspond to the points A-D in Fig. 3a and Fig. 4a. One can see a smoothing of the flow field within a given range $[K, K+1]$ (Figs. 5a and b, or Figs. 5c and d) and a sharp increase in the amplitudes both in the troposphere and the lower stratosphere, if Fig. 5b and Fig. 5c are compared. Note that, for all the cases considered, the strongest disturbances are located above the main topographic elevations, in accordance with the hydrostatic model prediction. 

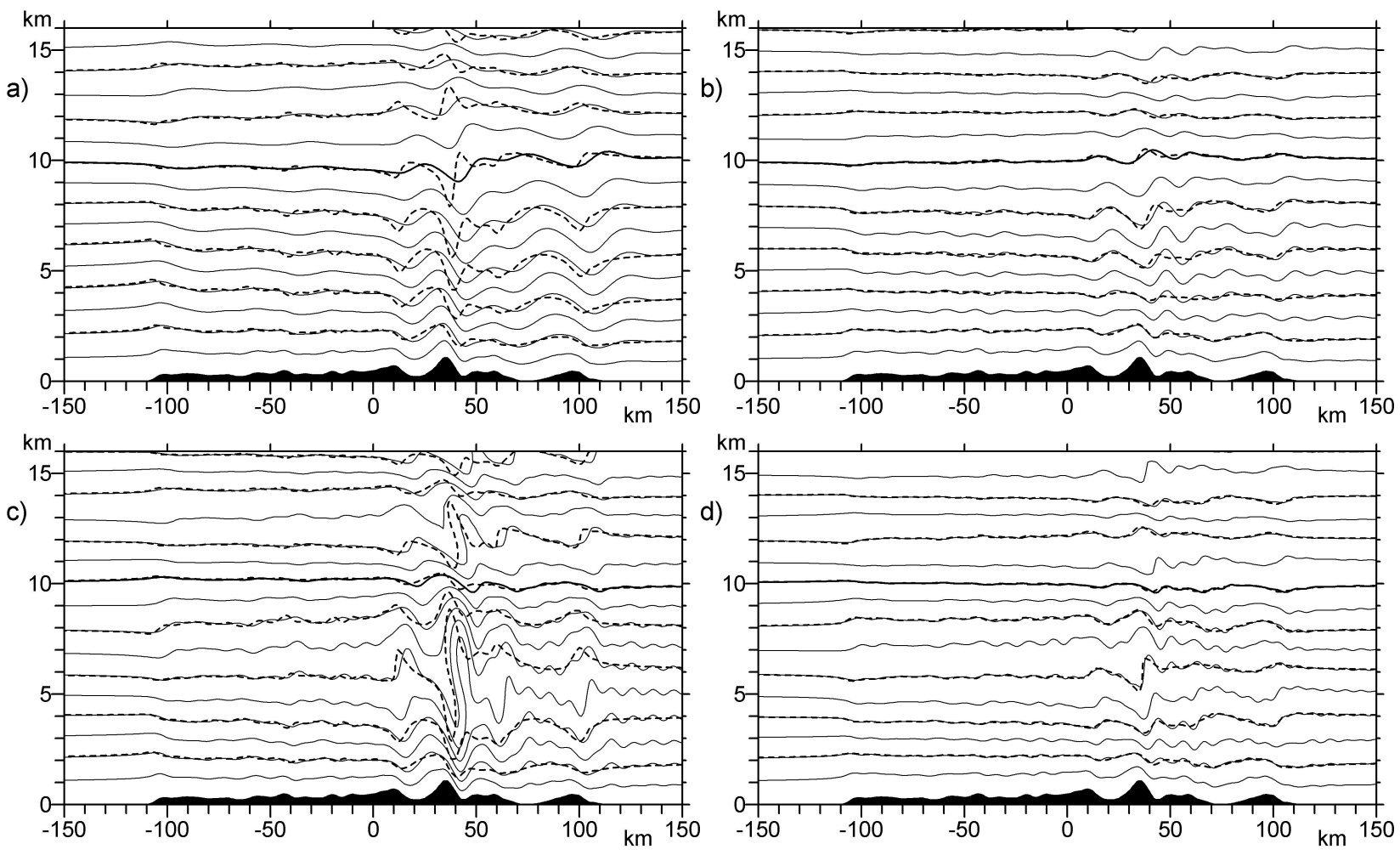

Fig. 5. Streamlines for hydrostatic solution, Eqs. $(29,30)$ (dashed), over the Ural Mountains, compared with non-hydrostatic solution, Eqs. $(14,15)$ (solid). The flow fields (a-d) correspond to the points A-D in Fig. 3a. The bold solid line denotes an internal dividing surface (tropopause). The individual streamlines for non-hydrostatic and hydrostatic solutions are given with 1-km and 2-km increments in their undisturbed height, correspondingly. Flow is directed from left to right.

The similar dependence of the flow field on the value of the reduced frequency was also obtained for the Scandinavian mountains. For all the reliefs the hydrostatic model predicts correct values of stream line vertical shifts but, of course, fails to predict a thin structure of the flow formed by short wave components of the resulting field. As an example, Figs. 5a and b shows the flow fields in case b for the first and second quasi-resonant peaks, correspondingly (points A and B in Figs. 3b and $4 b$ ). The flow in Fig. 5a corresponds to the troposphere having a thickness of one-half of the corresponding Lyra scale. This results in an overall downdraft in the middle and upper troposphere above the windward slope and the main ridge (see figure). In the vicinity of the second quasi-resonant peak, local regions of statically unstable flow persist over the main ridge in the middle troposphere and above the tropopause (compare Fig. 5c and Fig. 5b), as it takes place in the case of the Ural Mountains.

\section{Three-layer solution}

The solution given by Eqs. $(29,30)$ can be easily expanded to the multi-layer flow by means of the above described technique to approximately account for the lapse rate variation in the troposphere. It should be taken into account, however, that the flow disturbances in the troposphere can be sufficiently strong in a wide range of atmospheric parameters. Consequently, the usage of linearized conditions on the dividing streamlines, as discussed on page 2 , can lead to unpredictable errors in the resulting field, whose magnitude will accumulate as the number of layers increases. Thus, the results presented below should be treated as qualitative estimations of the real effects connected to the interaction of model layers.

Here we discuss the results of calculations in the framework of a three-layer model in which the troposphere is represented by two layers with an internal dividing surface placed at height $H_{t}<H$. The numerical experiments have shown a strong response to the tropopause height for such a model as well, with the hydrostatic component of the solution playing a dominant role in this phenomena, although considerable amplification of the flow disturbances associated with the variation of static stability in the troposphere can also take place. As an example, Fig. 7 shows the dependence of hydrostatic drag coefficient on the incident flow velocity in the case $\mathrm{b}$ for four different lapse rates $(9,8$, 7 , and $5 \mathrm{~K} / \mathrm{km})$ in the upper troposphere $\left(H_{t} \leq z \leq H\right)$, where $H_{t}=7 \mathrm{~km}$, with the same wind velocity range and lapse rates in the bottom and upper layers, as defined by Eq. (54), i.e. $\gamma=7 \mathrm{~K} / \mathrm{km}$ for $0 \leq z \leq H_{t}$, and $\gamma=0 \mathrm{~K} / \mathrm{km}$ for $z \geq H$. The bold, 

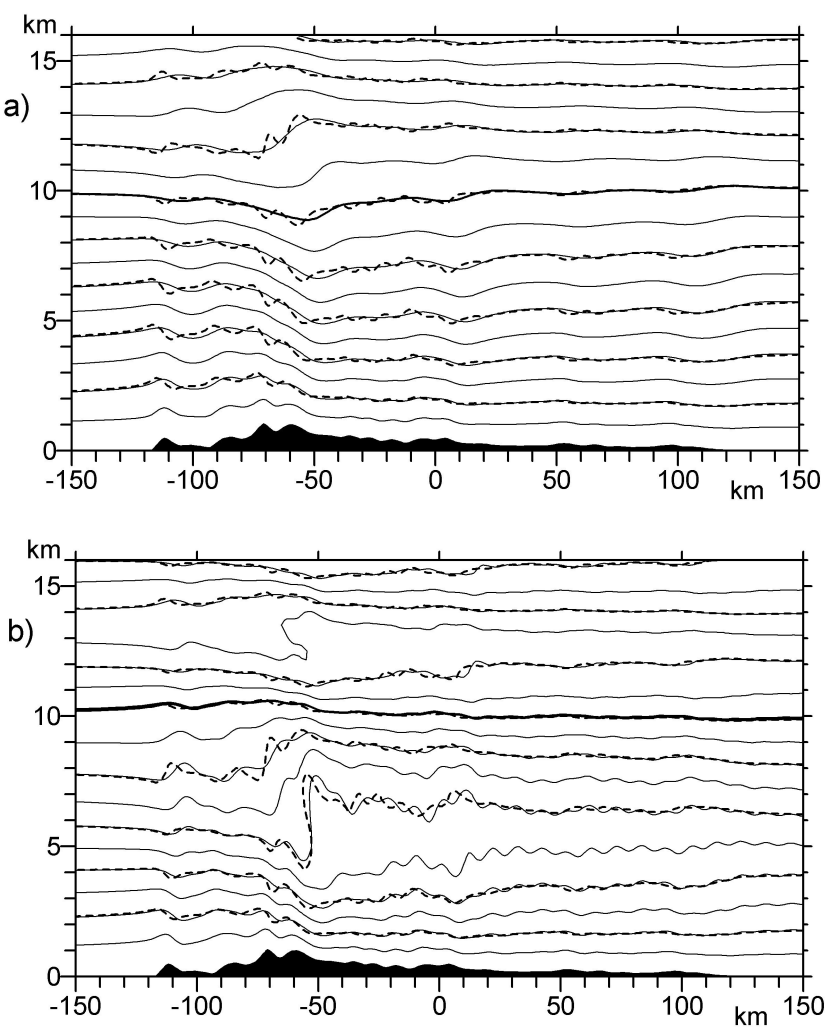

Fig. 6. Streamlines over the Scandinavian mountains, case b. Notations are the same as in Figs. 5a-d. The flow fields (a), (b) correspond to the points A, B in Fig. 3b. Flow is directed from left to right.

solid curve in Fig. 7 represents a solution with a constant lapse rate below the tropopause $(7 \mathrm{~K} / \mathrm{km})$, identical to that shown in Fig. 3b. According to Fig. 7, the increase in the lapse rate in the upper troposphere, lowering the static stability of the troposphere as a whole, leads to some decrease in the optimal values of $k$ at which maximal values of the drag coefficient are attained, in accordance with the linear condition given by Eq. (47).

As it has been already mentioned, for sufficiently low velocities $\left(U_{0} \leq 30 \mathrm{~ms}^{-1}\right)$, the major differences between the hydrostatic and non-hydrostatic solutions take place in the vicinity of the quasi-resonant peaks, where, along with the hydrostatic component of the solution, the amplification of the short, partially trapped waves takes place (Berkshire and Warren, 1970). One can see from Figs. 5 and 6, that this feature reveals itself as a train of partially trapped lee waves in the middle troposphere (whose horizontal lengths are close to the corresponding Lyra scale), as well as in a thin structure of the flow directly above the main tops. The magnitude of the short-wave component of a solution generally increases as the static stability in the upper troposphere decreases (Scorer, 1949; Berkshire, 1975), leading to a somewhat stronger overestimation of flow energetics by the hydrostatic model, comparing with the two-layer cases described above. Yet, the hydrostatic approximation seems to be valid for the qualitative

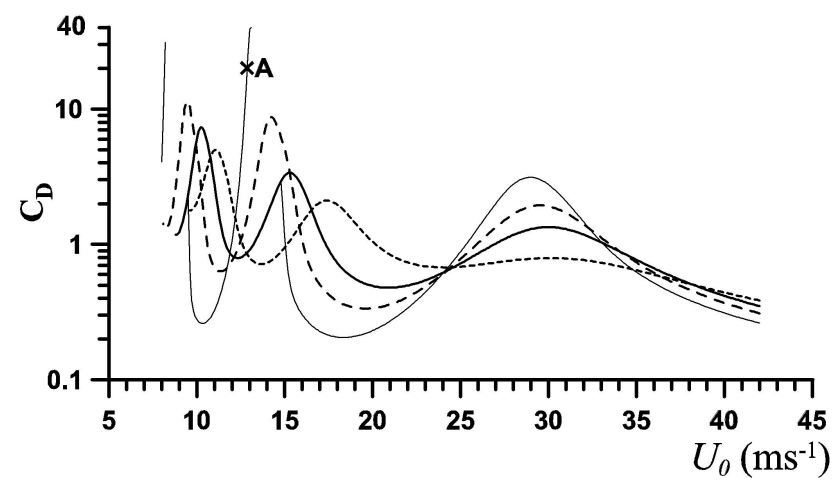

Fig. 7. Drag coefficient for the three-layer hydrostatic solution as a function of $U_{0}$ for the Scandinavian mountains, case $\mathrm{b}$, depending on the lapse rate in the middle layer $(7 \mathrm{~km} \leq z \leq 10 \mathrm{~km}): 9 \mathrm{~K} / \mathrm{km}$ (thin solid), $8 \mathrm{~K} / \mathrm{km}$ (long dashed), $7 \mathrm{~K} / \mathrm{km}$ (solid), and $5 \mathrm{~K} / \mathrm{km}$ (short dashed). The lapse rates in the bottom and upper layers are $7 \mathrm{~K} / \mathrm{km}$ and $0 \mathrm{~K} / \mathrm{km}$, correspondingly.

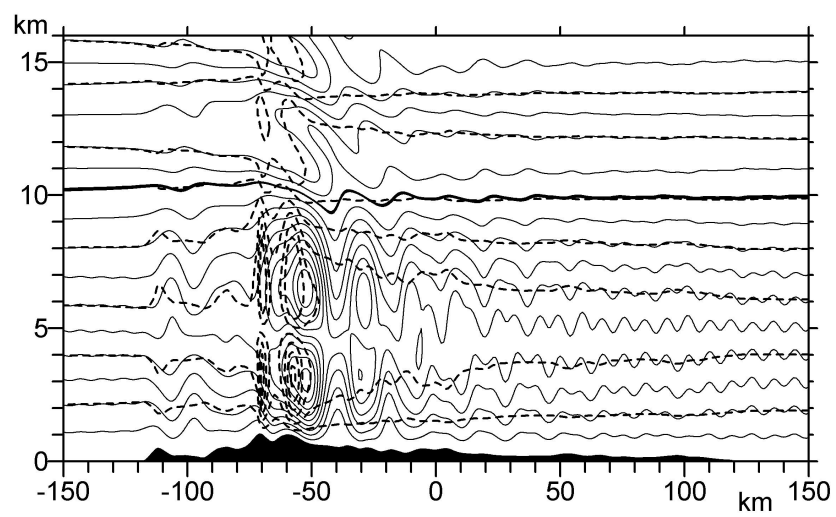

Fig. 8. Streamlines over the Scandinavian mountains, case b. Notations are the same as in Figs. 5a-d. The flow fields correspond to the point $\mathrm{A}$ in Fig. 7 near the second quasi-resonant peak of $C_{D}$. Flow is directed from left to right. The values of the drag coefficient are 20.0 and 9.8 for the hydrostatic and non-hydrostatic solutions, correspondingly.

analysis of such situations, as well, except for the cases in which the quasi-resonant effects are anomalously strong.

As an example of such a situation, the flow in the vicinity of the second quasi-resonant peak for $\gamma=9 \mathrm{~K} / \mathrm{km}$ in the upper troposphere (point A in Fig. 7) is shown in Fig. 8. Comparison of this flow with that shown in Fig. 5 brings to conclusion that it is the rotor motions above the most elevated part of the relief that plays the dominant role in the formation of exceptionally high values of the wave drag $(26.6 \mathrm{H} / \mathrm{m})$. In this example, the intensification of short waves leads not only to lee waves generation but also to very strong up- and downdrafts immediately above the ridge downstream from the most intense rotors - the features which are not reproduced by the hydrostatic model. As a consequence, the value of the hydrostatic drag coefficient (20.0) proves to be approximately two times higher compared to 
that predicted by the non-hydrostatic model (9.8). Actually, the hydrostatic model fails to operate in the vicinity of the second and third peaks (wind velocity ranges 13.1$14.7 \mathrm{~ms}^{-1}$ and $8.3-9.4 \mathrm{~ms}^{-1}$, correspondingly) because the further intensification of the rotor motions seen in Fig. 5 leads to an almost complete blocking of the incident flow throughout the troposphere. Evidently, the turbulence in the free atmosphere must play an exceptionally important role in such situations.

\section{Conclusions}

The results of the numerical calculations show a strong influence of the effects associated with the partial reflection of upward propagating wave energy by the tropopause on the flow field above the mesoscale mountains for typical atmospheric lapse rates and average wind velocities. In the case of a strong amplification the model predicts extensive statically unstable regions of decelerated flow located in the middle troposphere and the lower stratosphere, above the main topographic elevations, where a strong turbulence can be produced.

The hydrostatic approximation proves to be valid for quantitative evaluations of the wave drag, as well as for the prediction of statically unstable flow regimes in a wide range of atmospheric parameters, except for the case of exceptionally high wind velocities (expressed in terms of equivalent values of the reduced frequency as $k \leq 1$ ), as well as close to the local wave drag maxima, where its use can lead to substantial overestimation of the wave energy associated with short partially trapped waves. As a whole, the use of the linear approach leads to a significant (a 2-4 times) underestimation of the energetic characteristics of the overflow process for $k>2$, for typical mesoscale mountain systems. The finite height of the mountain can produce an appreciable effect on the conditions of flow amplification for sufficiently large $d$. The extreme values of wave drag have a tendency to shift to higher optimal values of $k$ (or height of the tropopause) compared to those predicted by linear analysis, as the dimensionless height $d$ of the obstacle increases.

The results of analytic theory are to be verified against experimental data which is the subject of further work.

Acknowledgements. The research was partially supported by the Russian Foundation for Basic Research under Grant 04-05-64723. I thank V. N. Kozhevnikov (Moscow State University) and V. M. Ponomarev (Obukhov Institute of Atmospheric Physics) for their comments and suggestions.

Topical Editor U.-P. Hoppe thanks a referee for his/her help in evaluating this paper.

\section{References}

Berkshire, F. H. and Warren, F. W. G.: Some aspects of linear lee wave theory for the stratosphere, Quart. J. Roy. Met. Soc., 96, 50-67, 1970.
Berkshire, F. H.: Two-dimensional linear lee wave modes for models including a stratosphere, Quart. J. Roy. Met. Soc., 101, 259266, 1975.

Blumen, W.: A random model of momentum flux by mountain waves, Geophys. Pub., 26, No. 2, 1-33, 1965.

Claus, A. J.: Large-amplitude motion of a compressible fluid in the atmosphere, J. Fluid. Mech., 19, 267-289, 1963.

Davies, H. C. and Phillips, P. D.: Mountain drag along the Gotthard section during ALPEX, J. Atmos. Sci., 42, 2093-2109, 1985.

Drazin, P. G. and Su, C. H.: A note on long-wave theory of airflow over a mountain, J. Atmos. Sci., 32, 437-439, 1975.

Durran, D. R.: Another look on downslope windstorms, Part I: The development of analogs to super-critical flow in an infinitely deep, continuously stratified flow, J. Atmos. Sci., 43, 2527-2543, 1986.

Durran, D. R.: Mountain waves and downslope winds. In: Atmospheric Processes over Complex Terrain, edited by: Blumen, W., Meteor. Monogr., Am. Met. Soc., 45, 59-81, 1990.

Eliassen, A. and Palm, E.: On the transfer of energy in stationary mountain waves, Geophys. Pub., 22, No. 3, 1-23, 1961.

Enell, C.-F., Steen, A., Wagner, T., Frie, U., Pfeilsticker, K., Platt, U., Fricke, K.-H.: Occurence of polar stratospheric clouds at Kiruna, Ann. Geophys., 17, 1457-1462, 1999,

SRef-ID: 1432-0576/ag/1999-17-1457.

Gutman, L. N.: An Introduction to the Nonlinear Theory of Mesoscale Meteorological Processes, Leningrad, 1969.

Huppert, H. E. and Miles, J. W.: Lee waves in stratified flow, 3. Semi-elliptical obstacle, J. Fluid Mech., 35, 481-496, 1969.

Klemp, J. B. and Lilly, D. K.: The Dynamics of Wave-Induced Downslope Winds, J. Atmos. Sci., 32, 320-339, 1975.

Klemp, J. B. and Lilly, D. K.: Numerical simulation of hydrostatic mountain waves, J. Atmos. Sci., 35, 78-107, 1978.

Kozhevnikov, V. N.: A review of the present state of the theory of mesoscale oragraphic inhomogeneities of the vertical current field, Tr. (Trans.) Tsent. Aero. Observ., 98, 1-22, 1970.

Kozhevnikov, V. N. and Bedanokov, M. K.: Nonlinear multi-layer model for flow over mountains of arbitrary shape, Izv. Atmos. Oceanic Phys., 29, 780-792, 1993.

Kozhevnikov, V. N. and Bedanokov, M. K.: Wave disturbances over the Crimean mountains: theory and observations, Izv. Atmos. Oceanic Phys., 34, 546-556, 1998.

Kozhevnikov, V. N.: Atmospheric disturbances over mountains, Moscow, Nauchnii Mir, 1999.

Kozhevnikov, V. N. and Moiseenko, K. B.: Simulation of the flow over a mountain range with height-varying free-stream characteristics, Izv. Atmos. Oceanic Phys., 40, 142-152, 2004.

Lilly, D. K.: A severe downslope windstorm and aircraft turbulence event induced by a mountain wave, J. Atmos. Sci., 35, 59-77, 1978.

Lilly, D. K. and Klemp, J. B.: The effects of terrain shape on nonlinear hydrostatic mountain waves, J. Fluid Mech., 95, 241-261, 1979.

Long, R. R.: Some aspects of the flow of stratified fluids. III. Continuous density gradients, Tellus, 7, 341-357, 1955.

Lyra, G.: Theorie der stationären Leewellenstromung in freien Atmosphäre, Z. Angew. Math. Und Mech., 23, 1-28, 1943.

Miles, J. W.: Lee waves in stratified flow, Part 1, Thin barrier, J. Fluid Mech., 32, 549-567, 1968.

Miles, J. W. and Huppert, H. E.: Lee waves in stratified flow, Part 2, Semi-circular obstacle, J. Fluid Mech., 33, 803-814, 1968.

Miles, J. W. and Huppert, H. E.: Lee waves in stratified flow, 4. Perturbation approximations, J. Fluid Mech., 35, 497-525, 1969. 
Olver, F. W. J.: Introduction to asymptotics and special functions, Academic Press, New York and London, 1974.

Palm, E. and Foldvik, A.: Contribution to the theory of twodimensional mountain waves, Geophys. Pub., 21, No. 6, 1-30, 1960.

Röttger, J.: ST radar observations of atmospheric waves over mountainous areas: a review, Ann. Geophys., 18, 750-765, 2000, SRef-ID: 1432-0576/ag/2000-18-750.

Queney, P., Corby, G. A., Gerbier, N., Koschmieder, H., and Zierep, J.: The Airflow Over Mountains, edited by: Alaka, M. A. and Geneva, WMO Tech. Note 34, 1960.

Scorer, R. S.: Theory of waves in the lee of mountains, Quart. J. Roy. Met. Soc., 75, 41-56, 1949.

Smith, R. B.: The steeping of hydrostatic mountain waves, J. Atmos. Sci., 34, 1634-1654, 1977.
Smith, R. B.: The influence of mountains on the atmosphere, Adv. Geophys., 21, 81-230, 1979.

Smith, R. B.: On severe downslope winds, J. Atmos. Sci., 42, 2597 2603, 1985.

Stebel, K., Hooper, D. A., and Kirkwood, S.: The generation and vertical propagation of mountain lee waves over Esrange $\left(67.53^{\circ} \mathrm{N}, 21.06^{\circ} \mathrm{E}\right)$ in northern Sweden, Proceedings of the Quadrennial Ozone Symposium, 3-8 July, Hokkaido University, Sapporo, Japan, 651-652, 2000.

Titchmarsh, E. C.: Introduction to the theory of Fourier integrals, Oxford University Press, 1948.

Verlan, A. F. and Sizikov, V. S.: Methods of solution of Integral Equations including Computational Programmes, Kiev, Naukova Dumka, 1978. 